Proceedings of the Edinburgh Mathematical Society (2008) 51, 265-272 (C)

DOI:10.1017/S001309150500132X Printed in the United Kingdom

\title{
SOME INEQUALITIES FOR THE GENERALIZED GRÖTZSCH FUNCTION
}

\author{
XIAOHUI ZHANG, GENDI WANG AND YUMING CHU \\ Department of Mathematics, Huzhou University, Huzhou 313000, \\ People's Republic of China (xhzhang@hutc.zj.cn)
}

(Received 21 September 2005)

\begin{abstract}
For $a \in\left(0, \frac{1}{2}\right]$ and $r \in(0,1)$, let $\mu_{a}(r)$ be the so-called generalized Grötzsch function which appears in Ramanujan's generalized modular equations. In this paper, several sharp inequalities for $\mu_{a}(r)$ are obtained and a conjecture on $\mu_{a}(r)$, which was presented by Qiu and Vuorinen in 1999, is proved.
\end{abstract}

Keywords: generalized elliptic integrals; generalized Grötzsch function; monotonicity; inequalities 2000 Mathematics subject classification: Primary 33E05; 33C05

\section{Introduction}

Throughout this paper, we let $r^{\prime}=\sqrt{1-r^{2}}$ for $r \in(0,1)$. For $x>0$, let

$$
\Gamma(x)=\int_{0}^{\infty} t^{x-1} \mathrm{e}^{-t} \mathrm{~d} t \quad \text { and } \quad \Psi(x)=\frac{\Gamma^{\prime}(x)}{\Gamma(x)}
$$

be the classical Euler gamma function and psi function, respectively. For real numbers $a, b$ and $c$ with $c \neq 0,-1,-2, \ldots$, the Gaussian hypergeometric function is defined by $[\mathbf{1}]$

$$
F(a, b ; c ; x)={ }_{2} F_{1}(a, b ; c ; x) \equiv \sum_{n=0}^{\infty} \frac{(a, n)(b, n)}{(c, n)} \frac{x^{n}}{n !} \quad \text { for }|x|<1 .
$$

Here $(a, 0)=1$ for $a \neq 0$, and $(a, n)$ is the shifted factorial function

$$
(a, n) \equiv a(a+1)(a+2) \cdots(a+n-1)=\frac{\Gamma(n+a)}{\Gamma(a)}
$$

for $n \in N \equiv\{k: k$ is a positive integer $\}$. For $r \in(0,1)$ and $a \in(0,1)$, the generalized elliptic integrals $($ cf. $[\mathbf{5}, \S 5.5])$ are defined as

$$
\left.\begin{array}{c}
\mathcal{K}_{a}=\mathcal{K}_{a}(r) \equiv \frac{1}{2} \pi F\left(a, 1-a ; 1 ; r^{2}\right), \\
\mathcal{K}_{a}^{\prime}=\mathcal{K}_{a}^{\prime}(r) \equiv \mathcal{K}_{a}\left(r^{\prime}\right), \\
\mathcal{K}_{a}(0)=\frac{1}{2} \pi, \quad \mathcal{K}_{a}(1)=\infty,
\end{array}\right\}
$$


and

$$
\left.\begin{array}{l}
\mathcal{E}_{a}=\mathcal{E}_{a}(r) \equiv \frac{1}{2} \pi F\left(a-1,1-a ; 1 ; r^{2}\right), \\
\mathcal{E}_{a}^{\prime}=\mathcal{E}_{a}^{\prime}(r) \equiv \mathcal{E}_{a}\left(r^{\prime}\right), \\
\mathcal{E}_{a}(0)=\frac{1}{2} \pi, \quad \mathcal{E}_{a}(1)=\frac{\sin (\pi a)}{2(1-a)} .
\end{array}\right\}
$$

In the particular case $a=\frac{1}{2}$, the functions $\mathcal{K}_{a}$ and $\mathcal{E}_{a}$ reduce to $\mathcal{K}(r)$ and $\mathcal{E}(r)$, respectively, which are the well-known complete elliptic integrals of the first and second kind, respectively (cf. [6]). By symmetry of $a$ and $b$ in (1.2), it is obvious that $\mathcal{K}_{a}=\mathcal{K}_{1-a}$ for $a \in\left(0, \frac{1}{2}\right]$. Hence, we may assume that $a \in\left(0, \frac{1}{2}\right]$ in the following. For $a \in\left(0, \frac{1}{2}\right]$ and $r \in(0,1)$, define the generalized Grötzsch function

$$
\mu_{a}(r) \equiv \frac{\pi}{2 \sin (\pi a)} \frac{\mathcal{K}_{a}^{\prime}(r)}{\mathcal{K}_{a}(r)}, \quad \mu(r)=\mu_{1 / 2}(r) .
$$

The function $\mu_{a}(r)$ plays a very important role in some fields of mathematics. For instance, it is indispensable in geometric function theory, quasiconformal theory and the theory of Ramanujan's modular equations (see $[\mathbf{2 - 4}, \mathbf{7}]$ ). In the general case $a \in\left(0, \frac{1}{2}\right)$, however, the known properties of $\mu_{a}(r)$ are fewer than those of $\mu(r)$, which is the modulus of the Grötzsch ring domain in the plane. One of the tasks for the study of the properties of $\mu_{a}(r)$ is to extend the known results for $\mu(r)$ to the function $\mu_{a}(r)$. On the other hand, the comparison of $\mu_{a}(r)$ and $\mu(r)$ will enable us to use the known bounds of $\mu(r)$ to give estimates for $\mu_{a}(r)$. In this paper we shall extend some well-known results for $\mu(r)$ to $\mu_{a}(r)$, and give bounds of $\mu_{a}(r)$ in terms of $\mu(r)$ as well as in terms of elementary functions. We shall also prove a conjecture concerning $\mu_{a}(r)[\mathbf{9}]$, and show some properties of $\mathcal{K}_{a}$.

\section{Main results}

We now state our main results. Our first result answers the question of whether the well-known identities $[\mathbf{2},(5.2)$ and (5.4)]

$$
2 \mu\left(\frac{2 \sqrt{r}}{1+r}\right) \equiv \mu(r), \quad \mu\left(\frac{1-r}{1+r}\right) \equiv 2 \mu\left(r^{\prime}\right)
$$

can be extended to $\mu_{a}(r)$.

Theorem 2.1.

(i) For each $r \in(0,1)$, the function

$$
f(a, r) \equiv 2 \mu_{a}\left(\frac{2 \sqrt{r}}{1+r}\right)-\mu_{a}(r)
$$

is strictly decreasing in a from $\left(0, \frac{1}{2}\right]$ onto $[0, \infty)$. In particular, for all $r \in(0,1)$ and $a \in\left(0, \frac{1}{2}\right]$,

$$
2 \mu_{a}\left(\frac{2 \sqrt{r}}{1+r}\right) \geqslant \mu_{a}(r)
$$

with equality if and only if $a=\frac{1}{2}$. 
(ii) For each $r \in(0,1)$, the function

$$
g(a, r) \equiv \mu_{a}\left(\frac{1-r}{1+r}\right)-2 \mu_{a}\left(r^{\prime}\right)
$$

is strictly increasing in a from $\left(0, \frac{1}{2}\right]$ onto $(-\infty, 0]$. In particular, for all $r \in(0,1)$ and $a \in\left(0, \frac{1}{2}\right]$,

$$
\mu_{a}\left(\frac{1-r}{1+r}\right) \leqslant 2 \mu_{a}\left(r^{\prime}\right)
$$

with equality if and only if $a=\frac{1}{2}$.

The next theorem gives comparisons of $\mu_{a}(r)$ and $\mu(r)$.

\section{Theorem 2.2.}

(i) For each $r \in(0,1)$, the function $h(a, r) \equiv a^{2} \mu_{a}(r)$ is strictly increasing in a from $\left(0, \frac{1}{2}\right]$ onto $\left(0, \frac{1}{4} \mu(r)\right]$.

(ii) For each $a \in\left(0, \frac{1}{2}\right]$, the function $H(r) \equiv \mu_{a}(r) / \mu(r)$ is strictly increasing from $(0,1)$ onto $\left(1,1 / \sin ^{2}(\pi a)\right)$. In particular,

$$
\mu(r) \leqslant \mu_{a}(r) \leqslant \frac{1}{\sin ^{2}(\pi a)} \mu(r)
$$

with equality in each instance if and only if $a=\frac{1}{2}$.

Applying Theorem 2.2, one can easily derive monotonicity properties of certain functions defined in terms of $\mu_{a}(r)$ and some elementary functions. As an example, we give the following theorem.

\section{Theorem 2.3.}

(i) The function

$$
f_{1}(r) \equiv \frac{\mu_{a}(r)}{\sqrt{r^{\prime}} \log (4 / r)}
$$

is strictly increasing from $(0,1)$ onto $(1, \infty)$.

(ii) The function

$$
f_{2}(r) \equiv \frac{\mu_{a}(r)}{\operatorname{arth} \sqrt[4]{r^{\prime}}}
$$

is strictly increasing from $(0,1)$ onto $(1, \infty)$.

(iii) The function $f_{3}(r) \equiv \mu_{a}(r)$ arth $\sqrt[4]{r}$ is strictly increasing from $(0,1)$ onto

$$
\left(0, \frac{\pi^{2}}{4 \sin ^{2}(\pi a)}\right)
$$

Remark 2.4. In [11, Theorem 1.14], the monotonicity properties of $f(a, r)$ and $g(a, r)$ as functions of $r \in(0,1)$, have been obtained, and [11, Theorem 1.22] says that the function $\mu_{a}(r)$ is strictly decreasing from $\left(0, \frac{1}{2}\right]$ onto $[\mu(r), \infty)$ with respect to $a$ for given $r$.

In [9], it was conjectured that the function $f_{2}(r) \equiv \mu_{a}(r) / \operatorname{arth} \sqrt[4]{r^{\prime}}$ is increasing from $(0,1)$ onto $(1, \infty)$. Theorem 2.3 (ii) gives the proof of this conjecture. 


\section{Preliminaries}

In this section, we establish the following technical lemma, which shows some properties of $\mathcal{K}_{a}$ and is needed in the proofs of the main theorems.

\section{Lemma 3.1.}

(i) For $x \geqslant 0$ and $a \in\left(0, \frac{1}{2}\right)$, let $b=1-a$. Then the function $f_{1}(x) \equiv \Psi(x+a)-\Psi(x+b)$ is strictly increasing and concave from $[0, \infty)$ onto $[\Psi(a)-\Psi(b), 0)$.

(ii) For each $n$, the function $f_{2}(a) \equiv(a, n)(1-a, n)$ is strictly increasing on $\left(0, \frac{1}{2}\right]$, while the function $g(a) \equiv f_{2}(a) / a$ is strictly decreasing on $\left(0, \frac{1}{2}\right]$.

(iii) For $a \in\left(0, \frac{1}{2}\right]$, the function

$$
f_{3}(r) \equiv \frac{\partial}{\partial a} \log \mathcal{K}_{a}(r)
$$

is strictly increasing on $(0,1)$.

(iv) The function $f_{4}(a) \equiv \mathcal{K}_{a} / a$ is strictly decreasing from $\left(0, \frac{1}{2}\right]$ onto $[2 \mathcal{K}(r), \infty)$.

(v) The function

$$
f_{5}(r) \equiv \frac{\mathcal{K}(r)}{\mathcal{K}_{a}(r)}
$$

is strictly increasing from $(0,1)$ onto $(1,1 / \sin (\pi a))$.

Proof. (i) It is well-known that $\Psi^{\prime}$ and $\Psi^{\prime \prime}$ are strictly decreasing and increasing, respectively, on $(0, \infty)$. Since $a<b, f_{1}^{\prime}(x)=\Psi^{\prime}(x+a)-\Psi^{\prime}(x+b)>0$, and $f_{1}^{\prime \prime}(x)=$ $\Psi^{\prime \prime}(x+a)-\Psi^{\prime \prime}(x+b)<0$. Thus, the result for $f_{1}$ follows.

(ii) It follows from (1.3) that

$$
f_{2}(a)=(a, n)(1-a, n)=\frac{\Gamma(n+a)}{\Gamma(a)} \frac{\Gamma(n+1-a)}{\Gamma(1-a)} .
$$

By logarithmic differentiation, we have

$$
f_{2}^{\prime}(a)=f_{2}(a)\left[f_{1}(n)-f_{1}(0)\right]
$$

which is a product of two positive functions by part (i). Hence, the monotonicity of $f_{2}$ follows.

By the reflection property of the gamma function $[\mathbf{1 2}]$,

$$
\Gamma(x) \Gamma(1-x)=\frac{\pi}{\sin (\pi x)},
$$

we get

$$
g(a)=\frac{\sin (\pi a)}{\pi a}[\Gamma(n+a) \Gamma(n+1-a)]
$$


Since

$$
\frac{\mathrm{d}}{\mathrm{d} a} \log [\Gamma(n+a) \Gamma(n+1-a)]=f_{1}(n)<0,
$$

$\Gamma(n+a) \Gamma(n+1-a)$ is strictly decreasing in $a$. Clearly, $\sin (\pi a) /(\pi a)$ is strictly decreasing in $a$ on $\left(0, \frac{1}{2}\right]$. Hence, the right-hand side of (3.1) is a product of two positive and strictly decreasing functions, so that $g$ is strictly decreasing in $a$.

(iii) By (1.2), we have

$$
f_{3}(r)=\frac{\sum_{n=0}^{\infty} A_{n} r^{2 n}}{\sum_{n=0}^{\infty} B_{n} r^{2 n}},
$$

where $A_{n}=f_{2}(a)\left[f_{1}(n)-f_{1}(0)\right] /(n !)^{2}$ and $B_{n}=f_{2}(a) /(n !)^{2}$. Since $A_{n} / B_{n}=f_{1}(n)-$ $f_{1}(0)$ is strictly increasing in $n$ by part (i), $f_{3}$ is strictly increasing in $r$ by [8, Lemma 2.1 .

(iv) By (1.2),

$$
f_{4}(a)=\frac{\pi}{2}\left[\sum_{n=0}^{\infty} \frac{g(a)}{(n !)^{2}} r^{2 n}\right],
$$

and hence the monotonicity of $f_{4}$ follows from part (ii). The limiting values are clear.

(v) Write

$$
C_{n}=\frac{\left(\frac{1}{2}, n\right)^{2}}{(a, n)(1-a, n)}
$$

Then

$$
\begin{aligned}
C_{n+1}-C_{n} & =\frac{\left(\frac{1}{2}, n\right)^{2}}{(a, n)(1-a, n)}\left[\frac{\left(\frac{1}{2}+n\right)^{2}}{(a+n)(1-a+n)}-1\right] \\
& =\frac{\left(\frac{1}{2}, n\right)^{2}}{(a, n)(1-a, n)} \frac{\frac{1}{4}-a(1-a)}{(a+n)(1-a+n)} \\
& >0
\end{aligned}
$$

and hence $C_{n}$ is strictly increasing in $n$. By (1.2),

$$
\frac{\mathcal{K}(r)}{\mathcal{K}_{a}(r)}=\sum_{n=0}^{\infty} \frac{\left(\frac{1}{2}, n\right)^{2}}{(n !)^{2}} r^{2 n}\left(\sum_{n=0}^{\infty} \frac{(a, n)(1-a, n)}{(n !)^{2}} r^{2 n}\right)^{-1}
$$

which is strictly increasing by $\left[\mathbf{8}\right.$, Lemma 2.1]. $f_{5}\left(0^{+}\right)=1$ is clear. By l'Hôpital's rule,

$$
f_{5}\left(1^{-}\right)=\lim _{r \rightarrow 1^{-}} \frac{\mathcal{E}-r^{\prime 2} \mathcal{K}}{2(1-a)\left(\mathcal{E}_{a}-r^{\prime 2} \mathcal{K}_{a}\right)}=\frac{1}{\sin (\pi a)} .
$$

This completes the proof of the lemma. 


\section{Proofs of main theorems}

Proof of Theorem 2.1. (i) Let $x=2 \sqrt{r} /(1+r)$. Then $x^{\prime}=(1-r) /(1+r), \mathrm{d} x / \mathrm{d} r=$ $x^{\prime} x / 2 r$.

Differentiation gives

$$
\begin{aligned}
\frac{\partial f}{\partial r} & =\frac{\mathrm{d}}{\mathrm{d} r}\left[2 \mu_{a}\left(\frac{2 \sqrt{r}}{1+r}\right)-\mu_{a}(r)\right] \\
& =-2 \frac{1}{x x^{\prime 2} F\left(a, 1-a ; 1 ; x^{2}\right)^{2}} \frac{x x^{\prime}}{2 r}+\frac{1}{r r^{\prime 2} F\left(a, 1-a ; 1 ; r^{2}\right)^{2}} \\
& =\frac{1}{r r^{\prime 2}}\left[\frac{1}{F\left(a, 1-a ; 1 ; r^{2}\right)^{2}}-(1+r)^{2} \frac{1}{F\left(a, 1-a ; 1 ; x^{2}\right)^{2}}\right] .
\end{aligned}
$$

By the Landen inequalities [10, Theorem 1.2] and Lemma 3.1 (iii), we have

$$
\begin{aligned}
\frac{\partial(\partial f / \partial r)}{\partial a} & =\frac{1}{r r^{\prime 2}}\left[-2 \frac{F_{a}^{\prime}\left(a, 1-a ; 1 ; r^{2}\right)}{F\left(a, 1-a ; 1 ; r^{2}\right)^{3}}+2(1+r)^{2} \frac{F_{a}^{\prime}\left(a, 1-a ; 1 ; x^{2}\right)}{F\left(a, 1-a ; 1 ; x^{2}\right)^{3}}\right] \\
& \geqslant \frac{2}{r r^{\prime 2}}\left[\frac{F_{a}^{\prime}\left(a, 1-a ; 1 ; x^{2}\right)}{F\left(a, 1-a ; 1 ; x^{2}\right) F\left(a, 1-a ; 1 ; r^{2}\right)^{2}}-\frac{F_{a}^{\prime}\left(a, 1-a ; 1 ; r^{2}\right)}{F\left(a, 1-a ; 1 ; r^{2}\right)^{3}}\right] \\
& =\frac{2}{r r^{\prime 2} F\left(a, 1-a ; 1 ; r^{2}\right)^{2}}\left[f_{3}(x)-f_{3}(r)\right] \\
& >0
\end{aligned}
$$

where

$$
F_{a}^{\prime}\left(a, 1-a ; 1 ; r^{2}\right)=\frac{\partial}{\partial a} F\left(a, 1-a ; 1 ; r^{2}\right) .
$$

It follows that $\partial f / \partial r$ is strictly increasing in $a \in\left(0, \frac{1}{2}\right)$. Hence, for $0<a<b \leqslant \frac{1}{2}$, we have

$$
\frac{\partial f(a, r)}{\partial r}<\frac{\partial f(b, r)}{\partial r}
$$

By integration,

$$
\int_{r}^{1} \frac{\partial f(a, r)}{\partial r} \mathrm{~d} r<\int_{r}^{1} \frac{\partial f(b, r)}{\partial r} \mathrm{~d} r
$$

hence,

$$
f(a, r)>f(b, r) .
$$

This yields the monotonicity of $f(a, r)$ in $a$.

For $r \in(0,1), f\left(\frac{1}{2}, r\right)=0$ by $(2.1)$. Write $A(a, n)=(a, n)(1-a, n) /(n !)^{2}$ and $B(a, n)=$ $\Gamma(n+a) \Gamma(n+1-a) /(n !)^{2}$. By $(1.2)$ and $(1.6)$,

$$
\begin{aligned}
f(a, r) & =2 \frac{\pi}{2 \sin (\pi a)} \frac{\sum_{n=0}^{\infty} A(a, n) x^{\prime 2 n}}{\sum_{n=0}^{\infty} A(a, n) x^{2 n}}-\frac{\pi}{2 \sin (\pi a)} \frac{\sum_{n=0}^{\infty} A(a, n) r^{\prime 2 n}}{\sum_{n=0}^{\infty} A(a, n) r^{2 n}} \\
& =\frac{\pi}{\sin (\pi a)}\left\{\frac{\sum_{n=0}^{\infty} \sin (\pi a) \pi^{-1} B(a, n) x^{\prime 2 n}}{\sum_{n=0}^{\infty} \sin (\pi a) \pi^{-1} B(a, n) x^{2 n}}-\frac{1}{2} \frac{\sum_{n=0}^{\infty} \sin (\pi a) \pi^{-1} B(a, n) r^{\prime 2 n}}{\sum_{n=0}^{\infty} \sin (\pi a) \pi^{-1} B(a, n) r^{2 n}}\right\}
\end{aligned}
$$




$$
\begin{aligned}
& =\frac{\pi}{\sin (\pi a)}\left\{\frac{1+\sin (\pi a) \pi^{-1} \sum_{n=1}^{\infty} B(a, n) x^{\prime 2 n}}{1+\sin (\pi a) \pi^{-1} \sum_{n=1}^{\infty} B(a, n) x^{2 n}}-\frac{1}{2} \frac{1+\sin (\pi a) \pi^{-1} \sum_{n=1}^{\infty} B(a, n) r^{\prime 2 n}}{1+\sin (\pi a) \pi^{-1} \sum_{n=1}^{\infty} B(a, n) r^{2 n}}\right\} \\
& \sim \frac{\pi}{2 \sin (\pi a)}(a \rightarrow 0),
\end{aligned}
$$

and hence $f\left(0^{+}, r\right)=\infty$. The inequality $(2.2)$ and its equality case are clear.

(ii) Let $t=(1-r) /(1+r)$. Then $r^{\prime}=2 \sqrt{t} /(1+t)$, and $g(a, r)=-f(a, t)$. Hence, the assertion about $g$ follows from part (i).

Proof of Theorem 2.2. (i) By [3, Theorem 4.1(5)], we have

$$
h_{r}(a, r) \equiv \frac{\mathrm{d} h}{\mathrm{~d} r}=-\frac{\pi^{2}}{4} \frac{a^{2}}{r r^{\prime 2} \mathcal{K}_{a}^{2}},
$$

which is strictly decreasing in $a$ by Lemma 3.1 (iv). Therefore, for $0<a<b \leqslant \frac{1}{2}$,

$$
\int_{r}^{1} h_{r}(a, r) \mathrm{d} r>\int_{r}^{1} h_{r}(b, r) \mathrm{d} r .
$$

This gives

$$
a^{2} \mu_{a}(r)<b^{2} \mu_{b}(r)
$$

and hence $h(a, r)$ is strictly increasing in $a$. Clearly, $h\left(\frac{1}{2}, r\right)=\frac{1}{4} \mu(r)$. Let $A(a, n)$ and $B(a, n)$ be as in the proof of Theorem 2.1(i). Then

$$
\begin{aligned}
h\left(0^{+}, r\right) & =\lim _{a \rightarrow 0^{+}} a^{2} \frac{\pi}{2 \sin (\pi a)} \frac{\sum_{n=0}^{\infty} A(a, n) r^{\prime 2 n}}{\sum_{n=0}^{\infty} A(a, n) r^{2 n}} \\
& =\lim _{a \rightarrow 0^{+}} a \frac{\pi a}{\sin (\pi a)} \frac{\sum_{n=0}^{\infty} \sin (\pi a) \pi^{-1} B(a, n) r^{\prime 2 n}}{\sum_{n=0}^{\infty} \sin (\pi a) \pi^{-1} B(a, n) r^{2 n}} \\
& =\lim _{a \rightarrow 0^{+}} a \frac{\pi a}{\sin (\pi a)} \frac{1+\sin (\pi a) \pi^{-1} \sum_{n=1}^{\infty} B(a, n) r^{\prime 2 n}}{1+\sin (\pi a) \pi^{-1} \sum_{n=1}^{\infty} B(a, n) r^{2 n}} \\
& =0 .
\end{aligned}
$$

(ii) Write $H_{1}(r)=\mu_{a}(r)$ and $H_{2}(r)=\mu(r)$. Then $H_{1}\left(1^{-}\right)=H_{2}\left(1^{-}\right)=0$ and, by $[\mathbf{3}$, Theorem 4.1(5)],

$$
\frac{H_{1}^{\prime}(r)}{H_{2}^{\prime}(r)}=\frac{-\frac{1}{4} \pi^{2}\left(r r^{\prime 2} \mathcal{K}_{a}^{2}\right)^{-1}}{-\frac{1}{4} \pi^{2}\left(r r^{\prime 2} \mathcal{K}^{2}\right)^{-1}}=\left(\frac{\mathcal{K}}{\mathcal{K}_{a}}\right)^{2}
$$

Hence, the monotonicity of $H$ follows from Lemma 3.1(v) and [3, Lemma 5.1].

The limiting values follow from l'Hôpital's rule, (4.1) and Lemma 3.1(v). The second inequality in (2.4) is clear, while the first inequality in (2.4) holds by [11, Theorem $1.22]$.

Proof of Theorem 2.3. Parts (i) and (ii) follow from Theorem 2.2 (ii) and the corresponding results for $\mu(r)$ (see [2, Theorem 5.13(5), (6)]). Part (iii) follows from part (ii) and the identity

$$
\mu_{a}(r) \mu_{a}\left(r^{\prime}\right)=\frac{\pi^{2}}{4 \sin ^{2}(\pi a)} .
$$


Acknowledgements. This research was partly supported by the National Science Foundation of China under Grant no. 10471039 and by the National Science Foundation of Zhejiang Province under Grant no. M103087.

The authors thank the referee for detailed comments, which led to some improvements in the text.

\section{References}

1. M. Abramowitz AND I. A. Stegun, Handbook of mathematical functions, with formulas, graphs, and mathematical tables (Dover, New York, 1966).

2. G. D. Anderson, M. K. Vamanamurthy and M. Vuorinen, Conformal invariants, inequalities, and quasiconformal maps (Wiley, 1997).

3. G. D. Anderson, S.-L. Qiu, M. K. Vamanamurthy and M. Vuorinen, Generalized elliptic integrals and modular equations, Pac. J. Math. 192 (2000), 1-37.

4. B. C. Berndt, S. Bhargava and F. G. Garvan, Ramanujan's theories of elliptic functions to alternative bases, Trans. Am. Math. Soc. 347 (1995), 4163-4244.

5. J. M. Borwein And P. B. Borwein, Pi and the AGM (Wiley, 1987).

6. F. Bowman, Introduction to elliptic functions with applications (Dover, New York, 1961).

7. R. KÜHNAU, Extremal functions in geometric function theory, higher transcendental functions, inequlities, in Handbook of complex analysis: geometric function theory (ed. R. Kühnau), Volume 2, pp. 661-668 (Elsevier, 2005).

8. S. Ponnusamy and M. Vuorinen, Asymptotic expansions and inequalities for hypergeometric functions, Mathematika 44 (1997), 278-301.

9. S.-L. QIU AND M. VUORINEN, Infinite products and normalized quotients of hypergeometric functions, SIAM J. Math. Analysis 30 (1999), 1057-1075.

10. S.-L. QIU AND M. Vuorinen, Landen inequalities for hypergeometric functions, Nagoya Math. J. 154 (1999), 31-56.

11. S.-L. QIU AND M. Vuorinen, Duplication inequalities for the ratios of hypergeometric functions, Forum Math. 12 (2000), 109-133.

12. E. T. Whittaker And G. N. Watson, A course of modern analysis, 4nd edn (Cambridge University Press, 1958). 